\title{
GFC-Robust Risk Management Strategies under the Basel Accord*
}

\author{
Michael McAleer \\ Econometric Institute \\ Erasmus School of Economics \\ Erasmus University Rotterdam \\ and \\ Tinbergen Institute \\ The Netherlands \\ and \\ Institute of Economic Research \\ Kyoto University \\ Japan \\ Juan-Ángel Jiménez-Martín \\ Department of Quantitative Economics \\ Complutense University of Madrid \\ Teodosio Pérez-Amaral \\ Department of Quantitative Economics \\ Complutense University of Madrid
}

Revised: October 2010

* For financial support, the first author wishes to thank the Australian Research Council, National Science Council, Taiwan, and the Japan Society for the Promotion of Science. The second and third authors acknowledge the financial support of the Ministerio de Ciencia y Tecnología and Comunidad de Madrid, Spain. 


\begin{abstract}
A risk management strategy is proposed as being robust to the Global Financial Crisis (GFC) by selecting a Value-at-Risk (VaR) forecast that combines the forecasts of different VaR models. The robust forecast is based on the median of the point VaR forecasts of a set of conditional volatility models. This risk management strategy is GFC-robust in the sense that maintaining the same risk management strategies before, during and after a financial crisis would lead to comparatively low daily capital charges and violation penalties. The new method is illustrated by using the S\&P500 index before, during and after the 2008-09 global financial crisis. We investigate the performance of a variety of single and combined VaR forecasts in terms of daily capital requirements and violation penalties under the Basel II Accord, as well as other criteria. The median VaR risk management strategy is GFC-robust as it provides stable results across different periods relative to other VaR forecasting models. The new strategy based on combined forecasts of single models is straightforward to incorporate into existing computer software packages that are used by banks and other financial institutions.
\end{abstract}

Key words and phrases: Value-at-Risk (VaR), daily capital charges, robust forecasts, violation penalties, optimizing strategy, aggressive risk management strategy, conservative risk management strategy, Basel II Accord, global financial crisis.

JEL Classifications: G32, G11, G17, C53, C22. 


\section{Introduction}

The Global Financial Crisis (GFC) of 2008-09 has left an indelible mark on economic and financial structures worldwide, and caused a generation of investors to wonder how things could have become so bad (see, for example, Borio (2008)). There have been many questions asked about whether appropriate regulations were in place, especially in the USA, to ensure the appropriate monitoring and encouragement of (possibly excessive) risk taking.

The Basel II Accord ${ }^{1}$ was designed to monitor and encourage sensible risk taking, using appropriate models of risk to calculate Value-at-Risk (VaR) and subsequent daily capital charges. VaR is defined as an estimate of the probability and size of the potential loss to be expected over a given period, and is now a standard tool in risk management. It has become especially important following the 1995 amendment to the Basel Accord, whereby banks and other Authorized Deposit-taking Institutions (ADIs) were permitted (and encouraged) to use internal models to forecast daily VaR (see Jorion (2000) for a detailed discussion). The last decade has witnessed a growing academic and professional literature comparing alternative modelling approaches to determine how to measure VaR, especially for large portfolios of financial assets.

The amendment to the initial Basel Accord was designed to encourage and reward institutions with superior risk management systems. A back-testing procedure, whereby actual returns are compared with the corresponding VaR forecasts, was introduced to assess the quality of the internal models used by ADIs. In cases where internal models led to a greater number of violations than could reasonably be expected, given the confidence level, the ADI is required to hold a higher level of capita (see Table 1 for the

\footnotetext{
${ }^{1}$ When the Basel I Accord was concluded in 1988, no capital requirements were defined for market risk. However, regulators soon recognized the risks to a banking system if insufficient capital were held to absorb the large sudden losses from huge exposures in capital markets. During the mid-90's, proposals were tabled for an amendment to the 1988 Accord, requiring additional capital over and above the minimum required for credit risk. Finally, a market risk capital adequacy framework was adopted in 1995 for implementation in 1998. The 1995 Basel I Accord amendment provides a menu of approaches for determining market risk capital requirements, ranging from a simple to intermediate and advanced approaches. Under the advanced approach (that is, the internal model approach), banks are allowed to calculate the capital requirement for market risk using their internal models. The use of internal models was introduced in 1998 in the European Union. The 26 June 2004 Basel II framework, implemented in many countries in 2008 (though not yet in the USA), enhanced the requirements for market risk management by including, for example, oversight rules, disclosure, management of counterparty risk in trading portfolios.
} 
penalties imposed under the Basel II Accord). Penalties imposed on ADIs affect profitability directly through higher capital charges, and indirectly through the imposition of a more stringent external model to forecast $\mathrm{VaR} .{ }^{2}$ This is one reason why financial managers may prefer risk management strategies that are passive and conservative rather than active and aggressive (for more on this, see below).

Excessive conservatism can have a negative impact on the profitability of ADIs as higher capital charges are subsequently required. Therefore, ADIs should perhaps consider a strategy that allows an endogenous decision as to how often ADIs should violate, and hence incur violation penalties, in any financial year (for further details, see McAleer and da Veiga (2008a, 2008b), McAleer (2009), Caporin and McAleer (2010a), and McAleer et al. (2009)). Additionally, ADIs need not restrict themselves to using only a single risk model. McAleer et al. (2009) propose a risk management strategy that consists in choosing from among different combinations of alternative risk models to forecast VaR. They discuss a combination of forecasts that was characterized as an aggressive strategy, and another that was regarded as a conservative strategy. ${ }^{3}$

Following such an approach, this paper suggests using a combination of VaR forecasts to obtain a crisis robust risk management strategy. The paper defines a crisis robust strategy as an optimal risk management strategy that remains unchanged regardless of whether it is used before, during or after a significant financial crisis. Parametric methods for forecasting VaR are typically fitted to historical returns assuming specific conditional distributions of returns, such as normality, Student-t, or generalized normal distribution. The VaR forecast depends on the parametric model, the conditional distribution and can be heavily affected by a few large observations. Some models provide many violations, but low daily capital charges. Additionally, these results can change drastically from tranquil to turbulent periods. In short, regardless of economic turbulence, is there a model to forecast $\mathrm{VaR}$ that provides a reasonable number of violations and daily capital charges?

\footnotetext{
${ }^{2}$ In the 1995 amendment (p. 16), a similar capital requirement system was recommended, but the specific penalties were left to each national supervisor. We consider that the penalty structure contained in Table 1 of this paper belongs only to Basel II, and was not part of Basel I or its 1995 amendment.

${ }^{3}$ This is a novel possibility. Technically, a combination of forecast models is also a forecast model. In principle, the adoption of a combination of forecast models by a bank is not forbidden by the Basel Accords, although it is subject to regulatory approval.
} 
We estimate several univariate conditional volatility models to forecast VaR, assuming different returns distributions (specifically, Gaussian, Student-t and Generalized Normal). Additionally, we present 12 new strategies based on combinations of stardard model VaR forecasts, namely: lowerbound, upperbound (as defined in McAleer et al. (2009)), the average, and nine additional strategies based on the $10^{\text {th }}, \ldots 50^{\text {th }}, \ldots 90^{\text {th }}$ percentiles. Models are compared over three different time periods to investigate whether we can find a risk management strategy that is robust over time (that is, crisisrobust). We provide evidence that using the median of the point VaR forecasts of a set of univariate conditional volatility models is a robust risk measure. A risk management strategy based on the median forecast is found to be superior to alternative single and composite model alternatives.

The remainder of the paper is organized as follows. In Section 2 we present the main ideas of the Basel II Accord Amendment as it relates to forecasting VaR and daily capital charges. Section 3 reviews some of the most well-known models of conditional volatility used to forecast VaR. In Section 4 the data used for estimation and forecasting are presented. Section 5 analyses the robust VaR forecasts before, during and after the 2008-09 GFC. Section 6 presents some conclusions.

\section{2. $\quad$ Forecasting Value-at-Risk and Daily Capital Charges}

The Basel II Accord stipulates that daily capital charges (DCC) must be set at the higher of the previous day's VaR or the average VaR over the last 60 business days, multiplied by a factor $(3+k)$ for a violation penalty, wherein a violation involves the actual negative returns exceeding the VaR forecast negative returns for a given day: ${ }^{4}$

$$
\mathrm{DCC}_{\mathrm{t}}=\sup \left\{-(3+\mathrm{k}) \overline{\mathrm{VaR}}_{60},-\mathrm{VaR}_{\mathrm{t}-1}\right\}
$$

where

\footnotetext{
${ }^{4}$ Our aim is to investigate the likely performance of the Basel II regulations. In this section we carry out our analysis applying the Basel II formulae to a period that includes the 2008-09 GFC, during which the Basel II Accord regulations were not fully implemented.
} 
$D C C_{t}=$ daily capital charges, which is the higher of $-(3+\mathrm{k}) \overline{\mathrm{VaR}}_{60}$ and $-\mathrm{VaR}_{\mathrm{t}-1}$,

$V a R_{t}=$ Value-at-Risk for day $t$

$\operatorname{VaR}_{t}=\hat{Y}_{t}-z_{t} \cdot \hat{\sigma}_{t}$

$\overline{\mathrm{VaR}}_{60}=$ mean $\mathrm{VaR}$ over the previous 60 working days,

$\hat{Y}_{t}=$ estimated return at time $\mathrm{t}$,

$z_{t}=1 \%$ critical value of the distribution of returns at time $\mathrm{t}$,

$\hat{\sigma}_{t}=$ estimated risk (or square root of volatility) at time $\mathrm{t}$,

$0 \leq \mathrm{k} \leq 1$ is the Basel II violation penalty (see Table 1 ).

\section{[Insert Table 1 here]}

The multiplication factor ${ }^{5}$ (or penalty), $k$, depends on the central authority's assessment of the ADI's risk management practices and the results of a simple backtest. It is determined by the number of times actual losses exceed a particular day's VaR forecast (Basel Committee on Banking Supervision (1996, 2006)). The minimum multiplication factor of 3 is intended to compensate for various errors that can arise in model implementation, such as simplifying assumptions, analytical approximations, small sample biases and numerical errors that tend to reduce the true risk coverage of the model (see Stahl (1997)). Increases in the multiplication factor are designed to increase the confidence level that is implied by the observed number of violations to the 99 per cent confidence level, as required by regulators (for a detailed discussion of VaR, as well as exogenous and endogenous violations, see McAleer (2009), Jiménez-Martin et al. (2009), and McAleer et al. (2009)).

In calculating the number of violations, ADIs are required to compare the forecasts of VaR with realised profit and loss figures for the previous 250 trading days. In 1995, the

\footnotetext{
${ }^{5}$ The formula in equation (1) is contained in the 1995 amendment to Basel I, while Table 1 appears for the first time in the Basel II Accord in 2004.
} 
1988 Basel Accord (Basel Committee on Banking Supervision (1988)) was amended to allow ADIs to use internal models to determine their VaR thresholds (Basel Committee on Banking Supervision (1995)). However, ADIs that propose using internal models are required to demonstrate that their models are sound. Movement from the green zone to the red zone arises through an excessive number of violations. Although this will lead to a higher value of $k$, and hence a higher penalty, violations will also tend to be associated with lower daily capital charges. ${ }^{6}$

Value-at-Risk refers to the lower bound of a confidence interval for a (conditional) mean, that is, a "worst case scenario on a typical day". If interest lies in modelling the random variable, $Y_{t}$, it could be decomposed as follows:

$$
Y_{t}=E\left(Y_{t} \mid F_{t-1}\right)+\varepsilon_{t}
$$

This decomposition states that $Y_{t}$ comprises a predictable component, $E\left(Y_{t} \mid F_{t-1}\right)$, which is the conditional mean, and a random component, $\varepsilon_{t}$. The variability of $Y_{t}$, and hence its distribution, is determined by the variability of $\varepsilon_{t}$. If it is assumed that $\varepsilon_{t}$ follows a conditional distribution, such that:

$$
\varepsilon_{t} \sim D\left(\mu_{t}, \sigma_{t}^{2}\right)
$$

where $\mu_{t}$ and $\sigma_{t}$ are the conditional mean and standard deviation of $\varepsilon_{t}$, respectively, these can be estimated using a variety of parametric, semi-parametric or non-parametric methods. The VaR threshold for $Y_{t}$ can be calculated as:

$$
\operatorname{VaR}_{t}=E\left(Y_{t} \mid F_{t-1}\right)-\alpha \sigma_{t},
$$

where $\alpha$ is the critical value from the distribution of $\varepsilon_{t}$ to obtain the appropriate confidence level. It is possible for $\sigma_{t}$ to be replaced by alternative estimates of the

\footnotetext{
${ }^{6}$ The number of violations in a given period is an important (though not the only) guide for regulators to approve a given VaR model.
} 
conditional standard deviation in order to obtain an appropriate VaR (for useful reviews of theoretical results for conditional volatility models, see Li et al. (2002) and McAleer (2005), who discusses a variety of univariate and multivariate, conditional, stochastic and realized volatility models).

Some recent empirical studies (see, for example, Berkowitz and O'Brien (2001), Gizycki and Hereford (1998), and Pérignon et al. (2008)) have indicated that some financial institutions overestimate their market risks in disclosures to the appropriate regulatory authorities, which can imply a costly restriction to the banks trading activity. ADIs may prefer to report high VaR numbers to avoid the possibility of regulatory intrusion. This conservative risk reporting suggests that efficiency gains may be feasible. In particular, as ADIs have effective tools for the measurement of market risk, while satisfying the qualitative requirements, ADIs could conceivably reduce daily capital charges by implementing a context-dependent market risk disclosure policy. For a discussion of alternative approaches to optimize $\mathrm{VaR}$ and daily capital charges, see McAleer (2008) and McAleer et al. (2009).

The next section describes several volatility models that are widely used to forecast the 1-day ahead conditional variances and VaR thresholds.

\section{Models for Forecasting VaR}

ADIs can use internal models to determine their VaR thresholds. There are alternative time series models for estimating conditional volatility. In what follows, we present several conditional volatility models to evaluate strategic market risk disclosure, namely GARCH, GJR and EGARCH, with normal, Student- $t$ and Generalized normal distribution errors, where the parameters are estimated.

These models are chosen as they are well known and widely used in the literature. For an extensive discussion of the theoretical properties of several of these models, see Ling and McAleer (2002a, 2002b, 2003a) and Caporin and McAleer (2010a). As an alternative to estimating the parameters, we also consider the exponential weighted 
moving average (EWMA) method by Riskmetrics (1996) and Zumbauch, (2007) that calibrates the unknown parameters. We include a section on these models to present them in a unified framework and notation, and to make explicit the specific versions we are using. Apart from EWMA, the models are presented in increasing order of complexity.

\subsection{GARCH}

For a wide range of financial data series, time-varying conditional variances can be explained empirically through the autoregressive conditional heteroskedasticity (ARCH) model, which was proposed by Engle (1982). When the time-varying conditional variance has both autoregressive and moving average components, this leads to the generalized $\operatorname{ARCH}(p, q)$, or $\operatorname{GARCH}(p, q)$, model of Bollerslev (1986). It is very common to impose the widely estimated $\operatorname{GARCH}(1,1)$ specification in advance.

Consider the stationary $\mathrm{AR}(1)-\mathrm{GARCH}(1,1)$ model for daily returns, $y_{t}$ :

$$
y_{t}=\varphi_{1}+\varphi_{2} y_{t-1}+\varepsilon_{t}, \quad\left|\varphi_{2}\right|<1
$$

for $t=1, \ldots, n$, where the shocks to returns are given by:

$$
\begin{aligned}
& \varepsilon_{t}=\eta_{t} \sqrt{h_{t}}, \quad \eta_{t} \sim \operatorname{iid}(0,1) \\
& h_{t}=\omega+\alpha \varepsilon_{t-1}^{2}+\beta h_{t-1},
\end{aligned}
$$

and $\omega>0, \alpha \geq 0, \beta \geq 0$ are sufficient conditions to ensure that the conditional variance $h_{t}>0$. The stationary AR(1)-GARCH(1,1) model can be modified to incorporate a nonstationary $\operatorname{ARMA}(\mathrm{p}, \mathrm{q})$ conditional mean and a stationary $\operatorname{GARCH}(\mathrm{r}, \mathrm{s})$ conditional variance, as in Ling and McAleer (2003b).

\section{$3.2 \quad$ GJR}

In the symmetric GARCH model, the effects of positive shocks (or upward movements in daily returns) on the conditional variance, $h_{t}$, are assumed to be the same as the 
negative shocks (or downward movements in daily returns). In order to accommodate asymmetric behaviour, Glosten, Jagannathan and Runkle (1992) proposed a model (hereafter GJR), for which GJR( $(1,1)$ is defined as follows:

$$
h_{t}=\omega+\left(\alpha+\gamma I\left(\eta_{t-1}\right)\right) \varepsilon_{t-1}^{2}+\beta h_{t-1}
$$

where $\omega>0, \alpha \geq 0, \alpha+\gamma \geq 0, \beta \geq 0$ are sufficient conditions for $h_{t}>0$, and $I\left(\eta_{t}\right)$ is an indicator variable defined by:

$$
I\left(\eta_{t}\right)= \begin{cases}1, & \varepsilon_{t}<0 \\ 0, & \varepsilon_{t} \geq 0\end{cases}
$$

as $\eta_{t}$ has the same sign as $\varepsilon_{t}$. The indicator variable differentiates between positive and negative shocks, so that asymmetric effects in the data are captured by the coefficient $\gamma$. For financial data, it is expected that $\gamma \geq 0$ because negative shocks have a greater impact on risk than do positive shocks of similar magnitude. The asymmetric effect, $\gamma$, measures the contribution of shocks to both short run persistence, $\alpha+\gamma / 2$, and to long run persistence, $\alpha+\beta+\gamma / 2$. Although GJR permits asymmetric effects of positive and negative shocks of equal magnitude on conditional volatility, the special case of leverage, whereby negative shocks increase volatility while positive shocks decrease volatility (see Black (1976) for an argument using the debt/equity ratio), cannot be accommodated (for further details on asymmetry versus leverage in the GJR model, see Caporin and McAleer (2010b)).

\subsection{EGARCH}

An alternative model to capture asymmetric behaviour in the conditional variance is the Exponential GARCH, or EGARCH(1,1), model of Nelson (1991), namely:

$$
\log h_{t}=\omega+\alpha\left|\frac{\varepsilon_{t-1}}{h_{t-1}}\right|+\gamma \frac{\varepsilon_{t-1}}{h_{t-1}}+\beta \log h_{t-1}, \quad|\beta|<1
$$


where the parameters $\alpha, \beta$ and $\gamma$ have different interpretations from those in the GARCH(1,1) and GJR(1,1) models.

EGARCH captures asymmetries differently from GJR. The parameters $\alpha$ and $\gamma$ in EGARCH(1,1) represent the magnitude (or size) and sign effects of the standardized residuals, respectively, on the conditional variance, whereas $\alpha$ and $\alpha+\gamma$ represent the effects of positive and negative shocks, respectively, on the conditional variance in GJR(1,1). Unlike GJR, EGARCH can accommodate leverage, depending on the restrictions imposed on the size and sign parameters.

As noted in McAleer et al. (2007), there are some important differences between EGARCH and the previous two models, as follows: (i) EGARCH is a model of the logarithm of the conditional variance, which implies that no restrictions on the parameters are required to ensure $h_{t}>0$; (ii) moment conditions are required for the GARCH and GJR models as they are dependent on lagged unconditional shocks, whereas EGARCH does not require moment conditions to be established as it depends on lagged conditional shocks (or standardized residuals); (iii) Shephard (1996) observed that $|\beta|<1$ is likely to be a sufficient condition for consistency of QMLE for EGARCH(1,1); (iv) as the standardized residuals appear in equation (7), $|\beta|<1$ would seem to be a sufficient condition for the existence of moments; and (v) in addition to being a sufficient condition for consistency, $|\beta|<1$ is also likely to be sufficient for asymptotic normality of the QMLE of $\operatorname{EGARCH}(1,1)$.

The three conditional volatility models given above are estimated under the following distributional assumptions on the conditional shocks: (1) normal, and (2) $t$, with estimated degrees of freedom. As the models that incorporate the $t$ distributed errors are estimated by QMLE, the resulting estimators are consistent and asymptotically normal, so they can be used for estimation, inference and forecasting.

\subsection{Exponentially Weighted Moving Average (EWMA)}


As an alternative to estimating the parameters of the appropriate conditional volatility models, Riskmetrics (1996) developed a model which estimates the conditional variances and covariances based on the exponentially weighted moving average (EWMA) method, which is, in effect, a restricted version of the $\mathrm{ARCH}(\infty)$ model. This approach forecasts the conditional variance at time $t$ as a linear combination of the lagged conditional variance and the squared unconditional shock at time $t-1$. The EWMA model calibrates the conditional variance as:

$$
h_{t}=\lambda h_{t-1}+(1-\lambda) \varepsilon_{t-1}^{2}
$$

where $\lambda$ is a decay parameter. Riskmetrics (1996) suggests that $\lambda$ should be set at 0.94 for purposes of analysing daily data. As no parameters are estimated, there are no moment or log-moment conditions.

\section{Data}

The data used for estimation and forecasting are the closing daily prices for Standard and Poor's Composite 500 Index (S\&P500), which were obtained from the Ecowin Financial Database for the period 3 January 2000 to 16 March 2010. Although it is unlikely that an ADI's typical market risk portfolio only tracks the S\&P500 index, it is used as an illustration of the broad movements of profits and losses of the equity portfolios of ADIs.

If $P_{t}$ denotes the market price, the returns at time $t\left(R_{t}\right)$ are defined as:

$$
R_{t}=\log \left(P_{t} / P_{t-1}\right)
$$

\section{[Insert Figures 1-2 and Table 2 here]}

Figure 1 shows the S\&P500 returns, for which the descriptive statistics are given in

Table 2. The extremely high positive and negative returns are evident from September 
2008 onward, and have continued well into 2009. The mean is close to zero, and the range is between $-9.5 \%$ and $+11 \%$. The Jarque-Bera Lagrange multiplier test rejects the null hypothesis of normally distributed returns. As the series displays high kurtosis, this would seem to indicate the existence of extreme observations, as can be seen in the histogram, which is not surprising for daily financial returns data.

Several measures of volatility are available in the literature. In order to gain some intuition, we adopt the measure proposed in Franses and van Dijk (1999), where the true volatility of returns is defined as:

$$
V_{t}=\left(R_{t}-E\left(R_{t} \mid F_{t-1}\right)\right)^{2}
$$

where $F_{t-1}$ is the information set at time $t-1$.

Figure 2 shows the S\&P500 volatility, as the square root of $V_{t}$ in equation (11). The series exhibit clustering that needs to be captured by an appropriate time series model. The volatility of the series appears to be high during the early $2000 \mathrm{~s}$, followed by a quiet period from 2003 to the beginning of 2007 . Volatility increases dramatically after August 2008, due in large part to the worsening global credit environment. This increase in volatility is even higher in October 2008. In less than four weeks in October 2008 , the S\&P500 index plummeted by $27.1 \%$. In less than three weeks in November 2008, starting the morning after the US elections, the S\&P500 index plunged a further 25.2\%. Overall, from late August 2008, US stocks fell by a scarcely unbelievable $42.2 \%$ to reach a low on 20 November 2008 .

An examination of daily movements in the S\&P500 index from 2000 suggests that large changes by historical standards are 4\% in either direction. From January 2000 to August 2008 , there was a $0.31 \%$ chance of observing an increase of $4 \%$ or more in one day, and a $0.18 \%$ chance of seeing a reduction of $4 \%$ or more in one day. Therefore, $99.5 \%$ of movements in the S\&P500 index during this period had daily swings of less than $4 \%$. Prior to September 2008, the S\&P500 index had only 7 days with massive 4\% gains, but since September 2008, there have been a further 12 such days. On the downside, before 
the current stock market meltdown, the S\&P500 index had only 4 days with huge 4\% or more losses whereas, during the recent panic, there were a further 17 such days.

This comparison is between more than 99 months and less than 6 months. During this short time span of financial panic, the $4 \%$ or more gain days chances increased 80 times while the chances of $4 \%$ or more loss days increased 32 times. Such movements in the S\&P500 index are unprecedented.

\section{Robust Forecasting of VaR and Evaluation Framework}

As seen in McAleer et al. (2010), the global financial crisis has affected the best risk management strategies by changing the optimal model for minimizing daily capital charges. The objective here is to provide a robust risk management strategy, namely one that does not change over time, even in the context of a GFC. This robust risk management strategy also has to lead to daily capital charges that are not excessive, and violation frequencies that are compatible with the Basel II Accord.

ADIs need not restrict themselves to using only a single risk model. We propose a risk management strategy that consists in choosing a forecast from among different combinations of alternative risk models to forecast VaR. McAleer et al. (2010) developed a risk management strategy that used combinations of several models for forecasting VaR. It was found that an aggressive risk management strategy (namely, choosing the supremum of VaR forecasts, or upperbound) yielded the lowest mean capital charges and largest number of violations. On the other hand, a conservative risk management strategy (namely, by choosing the infinum, or lowerbound) had far fewer violations, and correspondingly higher mean daily capital charges.

In this paper, we forecast $\mathrm{VaR}$ using combinations of the forecasts of individual VaR models, namely the rth percentile of the VaR forecasts of a set of univariate conditional volatility models. Alternative single models with different error distributions and several combinations are compared over three different time periods to investigate which, if any, of the risk management strategies may be robust. 
We conduct an exercise to analyze the performance of existing VaR forecasting models, as permitted under the Basel II framework, when applied to the S\&P500 index. Additionally, we analyze twelve new strategies based on combinations of the previous standard single-model forecasts of VaR, namely: lowerbound ( $0^{\text {th }}$ percentile), upperbound $\left(100^{\text {th }}\right.$ percentile), average, and nine additional strategies based on the $10^{\text {th }}$ through to the $90^{\text {th }}$ percentiles. It is intended to determine whether we can select a robust VaR forecast irrespective of the time period, and to provide reasonable daily capital charges and number of violation penalties.

\subsection{Evaluating Crisis-Robust Risk Management Strategies}

In Table 3 the performance of the different VaR forecasting models is evaluated using several standard criteria that are relevance for the risk manager, namely: daily capital charges (DCC), number of violations (NoV), the failure rate (that is, the ratio of NoV to number of days) (FailRa), accumulated losses ${ }^{7}$ (AcLoss), and the value of the asymmetric linear tick loss function ${ }^{8}$ (AlTick) that allows a comparison of model performance. As Giacomini and Komunjer (2005) argue, the tick loss function is the implicit loss function whenever the target is a forecast of a particular $\alpha$-quantile. This loss function takes into account the magnitude of the implicit cost associated with VaR forecasting errors. As VaR estimates are used frequently to assist in strategic financial decision-making and managing market risk, $\mathrm{VaR}$ forecasting errors can imply financial distress, such as incorrectly estimating daily capital charges subject to regulatory control.

The performance criteria are calculated for each model and error distribution, and for each of the three sub-samples, before, during, and after the 2008-09 GFC, where before is from 2 January 2008 to 11 August 2008, during is from 12 August 2008 to 9 March 2009, and after is from 10 March 2009 to 16 March 2010. Table 3 shows the

\footnotetext{
${ }^{7}$ López (1999) suggested measuring the accuracy of the VaR forecast on the basis of the distance between the observed returns and the forecasted $\mathrm{VaR}$ values if a violation occurs:

$\Psi_{t+1}=\left\{\begin{array}{c}\left|\mathrm{R}_{t+1}-V a \mathrm{R}_{t+1 \mid t}\right| \text { if } \mathrm{R}_{t+1}<0 \text { and } \mathrm{R}_{t+1}<V a \mathrm{R}_{t+1 \mid t} \text { otherwise } \\ 0\end{array}\right.$, a preferred VaR model is the one that minimizes the total loss value, $\Psi_{t+1}=\sum_{t=1}^{T} \Psi_{t}$.

${ }^{8}$ The tick loss function of order $\alpha$ defined as $L_{\alpha}\left(e_{t+1}\right)=\left(\alpha-1\left(e_{t+1}<0\right)\right) e_{t+1}$, where $e_{t+1}=R_{t+1}-V a R_{t+1}$.
} 
performance statistics for comparing the VaR forecasts for each sub-period. We also include 12 new forecasting strategies based on combinations of individual model VaR forecasts, namelt : lowerbound, upperbound, average, and nine additional strategies based on the $10^{\text {th }}$ through to the $90^{\text {th }}$ percentiles, including the median.

No risk model is found always to be superior to its competitors as there is no strategy that optimizes every evaluation statistic for the three sub-periods. Nonetheless, the $50^{\text {th }}$ percentile strategy (namely, the median - shaded row of Table 3) is found to be robust, as it produces adequate $\mathrm{VaR}$ forecasts that exhibit stable results across different periods relative to the other risk models. Furthermore, we compute for each sub-sample and each statistic the values of the $1^{\text {st }}, 2^{\text {nd }}$ and $3^{\text {rd }}$ quartiles. In Table 4 , the median strategy statistics fall in almost all cases in the 2nd quartile. The EGARCH-t model performance is close to the median strategy, except that the former has average daily capital charges, (AvDCC) slightly higher for the three periods. In general, the median strategy provides a robust $\mathrm{VaR}$ forecast, regardless of whether there is a GFC.

\section{[Insert Tables 3-4 here]}

As can be seen in Table 3, before the GFC, AvDCC based on the Riskmetrics VaR forecast is lower $(9.03 \%)$ than that based on the median $(9.77 \%)$. During the GFC, AvDCC based on the median strategy increased drastically (20.57\%), although not as much as when the Riskmetrics model is used (22.51\%). Finally, after the GFC, AvDCC decreased, becoming smaller for the median strategy (10.95\%) than for the Riskmetrics model (11.19\%).

Figure 3 shows the evolution of the evaluation statistics for the strategies based on the $10^{\text {th }}$ through to the $90^{\text {th }}$ percentiles in the three sub-samples. GFC-robustness, in our context, implies a trade-off between the number of violations and daily cost of capital charges. In general, when the chosen percentile increases, it is found that DCC decreases and NoV increases, all the more so when the selected percentile is greater than the median.

Figure 4 shows VaR forecasts using four models (Riskmetrics, median, upperbound and lowerbound) and S\&P500 returns, where the vertical axis represents returns, and the 
horizontal axis represents the period from 2 January 2008 to 16 March 2010. The S\&P500 returns are given as the upper blue line that fluctuates around zero. The upper red line represents the supremum of the VaR calculated for the individual volatility models, which reflects an aggressive risk management strategy, whereas the lower green line represents the infimum of the VaR calculated for the individual volatility models, which reflects a conservative risk management strategy. These two lines correspond to a combination of alternative risk models. The brown line is the median of the VaR, calculated for the individual models of volatility. As a benchmark, the black line denotes the Riskmetrics model.

\section{[Insert Figures 3-4 here]}

As can be seen in Figure 4, $\mathrm{VaR}$ forecasts obtained from the different models of volatility have fluctuated, as expected, during the first few months of 2008. It has been relatively low, at below 5\%, and relatively stable between April and August 2008. Around September 2008, VaR started increasing until it peaked in October 2008, between $10 \%$ and $15 \%$, which is almost a four-fold increase in VaR in a matter of one and a half months. In the last two months of 2008 , VaR decreased to values between $5 \%$ and $8 \%$, which is still twice as large as it had been just a few months earlier. Therefore, volatility has increased substantially during the GFC, and has remained relatively high thereafter.

Figure 5 includes daily capital charges based on the median VaR for the previous 60 days, which are the lower lines. The red line corresponds to the aggressive risk management strategy based on the supremum of the daily VaR forecasts of the alternative models of volatility, the green line corresponds to the conservative risk management strategy based on the infinum of the $\mathrm{VaR}$ forecasts, and the brown line denotes the robust strategy based on the median of the VaR forecasts. As a benchmark, we include DCC based on the Riskmetrics model. Table 3 shows that the Riskmetrics AvDCC and FailRa are higher than the median AvDCC, except for before the GFC. This can be seen in Figure 5 when comparing the DCC Riskmetrics and DCC median lines. The DCC median line is close to the upperbound DCC line virtually throughout the sample, but FailRa for the median strategy is always much lower and is falling within the Basel Accord limits than when the upperbound strategy is considered. 


\section{[Insert Figure 5 here]}

\section{Conclusion}

In this paper we proposed robust risk forecasts that use combinations of several conditional volatility models for forecasting VaR. Different strategies for combining models were compared over three different time periods, using S\&P500 to investigate whether we can determine a GFC-robust risk management strategy. Backtesting provided evidence that a risk management strategy based on VaR forecast corresponding to the $50^{\text {th }}$ percentile (median) of the $\mathrm{VaR}$ forecasts of a set of univariate conditional volatility models is robust, in that it yields reasonable daily capital charges, number of violations that do not jeopardize institutions that might use it, and more importantly, is invariant before, during and after the 2008-09 GFC.

It is worth noting that, as in McAleer et al. (2010), the VaR model minimizing DCC before, during and after the GFC changed frequently. Although the median model is not derived as the best model for minimizing DCC and the number of violation penalties, it is nevertheless a model that balances daily capital charges and violation penalties in minimizing DCC.

The idea of combining different VaR forecasting models is entirely within the spirit of the Basel II Accord, although its use would require approval by the regulatory authorities, as for any forecasting model. This approach is not computationally demanding, even though several models have to be specified and estimated over time. Further research is needed to compute the standard errors of the forecasts of the combination models, including the median forecast. 


\section{References}

Basel Committee on Banking Supervision, (1988), International Convergence of Capital Measurement and Capital Standards, BIS, Basel, Switzerland.

Basel Committee on Banking Supervision, (1995), An Internal Model-Based Approach to Market Risk Capital Requirements, BIS, Basel, Switzerland.

Basel Committee on Banking Supervision, (1996), Supervisory Framework for the Use of "Backtesting" in Conjunction with the Internal Model-Based Approach to Market Risk Capital Requirements, BIS, Basel, Switzerland.

Basel Committee on Banking Supervision, (2006), International Convergence of Capital Measurement and Capital Standards, a Revised Framework Comprehensive Version, BIS, Basel, Switzerland.

Berkowitz, J. and J. O'Brien (2001), How accurate are value-at-risk models at commercial banks?, Discussion Paper, Federal Reserve Board.

Black, F. (1976), Studies of stock market volatility changes, in 1976 Proceedings of the American Statistical Association, Business and Economic Statistics Section, pp. 177-181.

Bollerslev, T. (1986), Generalised autoregressive conditional heteroscedasticity, Journal of Econometrics, 31, 307-327.

Borio, C. (2008), The financial turmoil of 2007-?: A preliminary assessment and some policy considerations, BIS Working Papers No 251, Bank for International Settlements, Basel, Switzerland.

Caporin, M. and M. McAleer (2010a), The Ten Commandments for managing investments, Journal of Economic Surveys, 24, 196-200.

Caporin, M. and M. McAleer (2010b), Model selection and testing of conditional and stochastic volatility models, to appear in L. Bauwens, C. Hafner and S. Laurent (eds.), Handbook on Financial Engineering and Econometrics: Volatility Models and Their Applications, Wiley, New York (Available at SSRN: http://ssrn.com/abstract=1676826).

Engle, R.F. (1982), Autoregressive conditional heteroscedasticity with estimates of the variance of United Kingdom inflation, Econometrica, 50, 987-1007.

Franses, P.H. and D. van Dijk (1999), Nonlinear Time Series Models in Empirical Finance, Cambridge, Cambridge University Press. 
Giacomini, R., and Komunjer, I. (2005), Evaluation and combination of conditional quantile forecasts. Journal of Business \& Economic Statistics, 23, 416-431.

Gizycki, M. and N. Hereford (1998), Assessing the dispersion in banks' estimates of market risk: the results of a value-at-risk survey, Discussion Paper 1, Australian Prudential Regulation Authority.

Glosten, L., R. Jagannathan and D. Runkle (1992), On the relation between the expected value and volatility of nominal excess return on stocks, Journal of Finance, 46, 1779-1801.

Jimenez-Martin, J.-A., McAleer, M. and T. Pérez-Amaral (2009), The Ten Commandments for managing value-at-risk under the Basel II Accord, Journal of Economic Surveys, 23, 850-855.

Jorion, P. (2000), Value at Risk: The New Benchmark for Managing Financial Risk, McGraw-Hill, New York.

Li, W.K., S. Ling and M. McAleer (2002), Recent theoretical results for time series models with GARCH errors, Journal of Economic Surveys, 16, 245-269. Reprinted in M. McAleer and L. Oxley (eds.), Contributions to Financial Econometrics: Theoretical and Practical Issues, Blackwell, Oxford, 2002, pp. 933.

Ling, S. and M. McAleer (2002a), Stationarity and the existence of moments of a family of GARCH processes, Journal of Econometrics, 106, 109-117.

Ling, S. and M. McAleer (2002b), Necessary and sufficient moment conditions for the $\operatorname{GARCH}(r, s)$ and asymmetric power GARCH(r, s) models, Econometric Theory, $18,722-729$.

Ling, S. and M. McAleer, (2003a), Asymptotic theory for a vector ARMA-GARCH model, Econometric Theory, 19, 278-308.

Ling, S. and M. McAleer (2003b), On adaptive estimation in nonstationary ARMA models with GARCH errors, Annals of Statistics, 31, 642-674.

Lopez, J. A., (1999), "Methods for evaluating value-at-risk estimates," Economic Review, Federal Reserve Bank of San Francisco, pages 3-17.

McAleer, M. (2005), Automated inference and learning in modeling financial volatility, Econometric Theory, 21, 232-261.

McAleer, M. (2009), The Ten Commandments for optimizing value-at-risk and daily capital charges, Journal of Economic Surveys, 23, 831-849. 
McAleer, M., F. Chan and D. Marinova (2007), An econometric analysis of asymmetric volatility: theory and application to patents, Journal of Econometrics, 139, 259284.

McAleer, M., J.-Á. Jiménez-Martin and T. Pérez-Amaral (2009), A decision rule to minimize daily capital charges in forecasting value-at-risk, to appear in Journal of Forecasting (Available at SSRN: http://ssrn.com/abstract=1349844).

McAleer, Michael, J-A., Jiménez-Martin and T. Perez Amaral (2010), Has the Basel II Accord Encouraged Risk Management During the 2008-09 Financial Crisis?. (Available at SSRN: http://ssrn.com/abstract=1397239).

McAleer, M. and B. da Veiga (2008a), Forecasting value-at-risk with a parsimonious portfolio spillover GARCH (PS-GARCH) model, Journal of Forecasting, 27, 1-19.

McAleer, M. and B. da Veiga (2008b), Single index and portfolio models for forecasting value-at-risk thresholds, Journal of Forecasting, 27, 217-235.

Nelson, D.B. (1991), Conditional heteroscedasticity in asset returns: a new approach, Econometrica, 59, 347-370.

Pérignon, C., Z.Y. Deng and Z.J. Wang (2008), Do banks overstate their value-at-risk?, Journal of Banking \& Finance, 32, 783-794.

Riskmetrics (1996), J.P. Morgan Technical Document, $4^{\text {th }}$ Edition, New York, J.P. Morgan.

Shephard, N. (1996), Statistical aspects of ARCH and stochastic volatility, in O.E. Barndorff-Nielsen, D.R. Cox and D.V. Hinkley (eds.), Statistical Models in Econometrics, Finance and Other Fields, Chapman \& Hall, London, 1-67.

Stahl, G. (1997), Three cheers, Risk, 10, pp. 67-69.

Zumbauch, G. (2007), A Gentle Introduction to the RM 2006 Methodology, New York, Riskmetrics Group. 
Figure 1. Daily Returns on S\&P500 Index

3 January 2000 - 16 March 2010

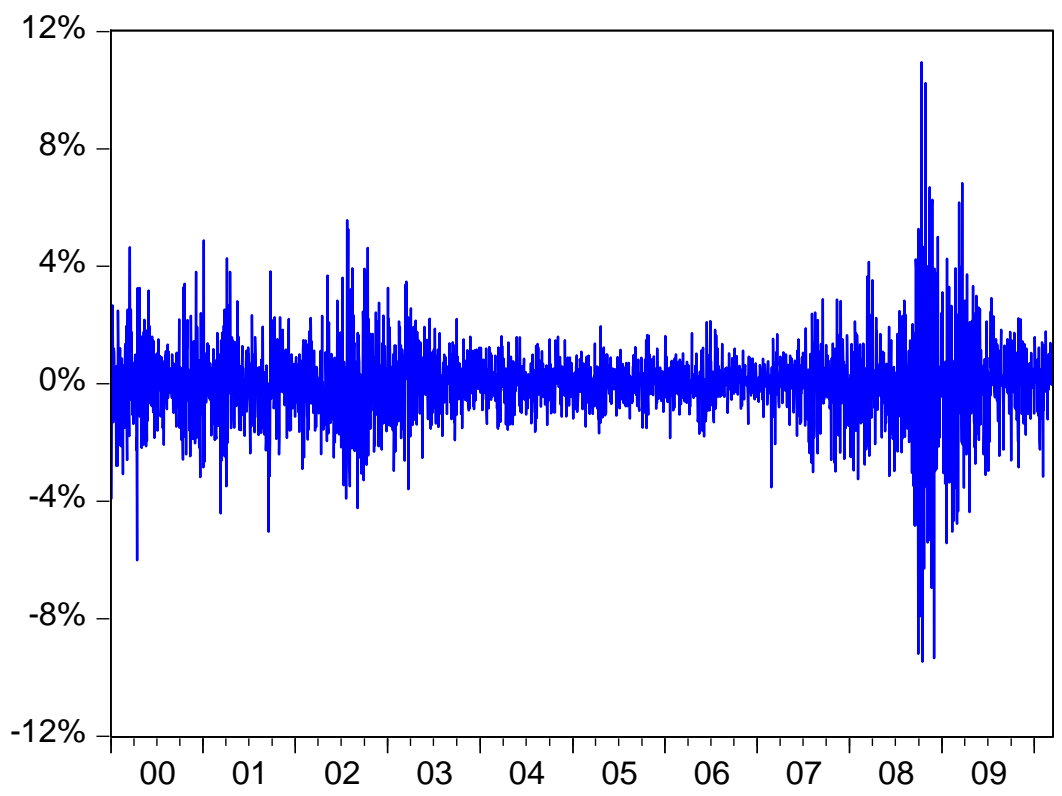

Figure 2. Daily Volatility in S\&P500 Returns

3 January 2000 - 16 March 2010

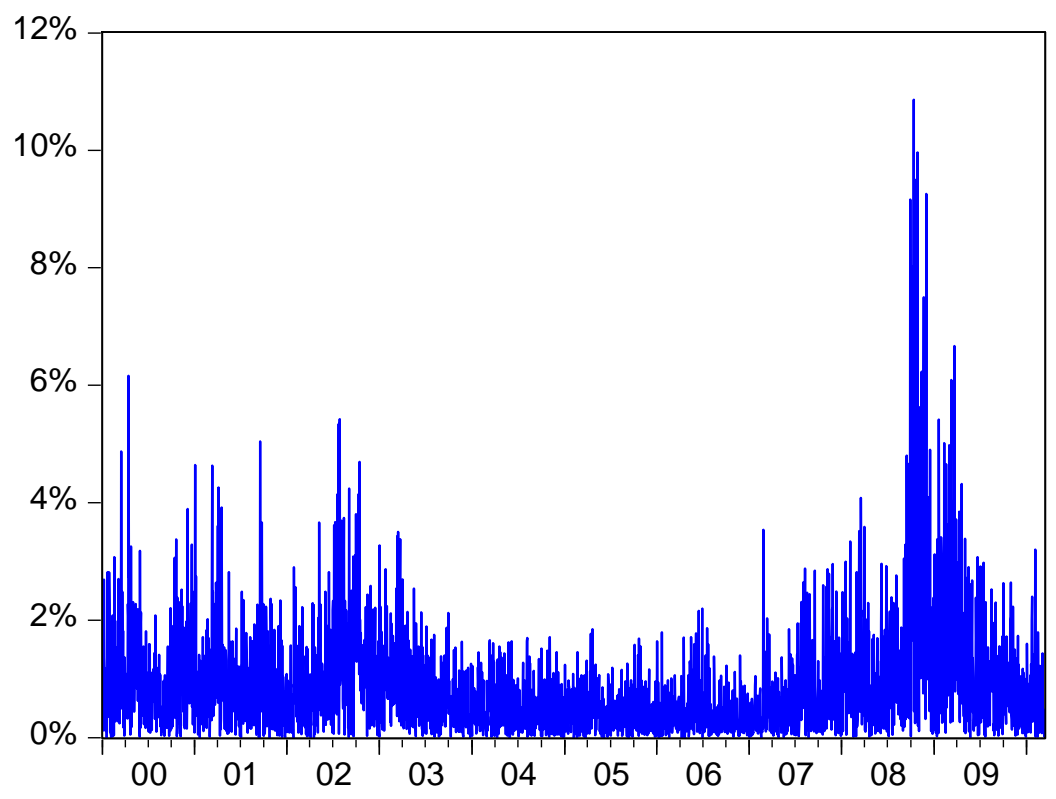


Figure 3. Criteria for Comparing Percentile Strategies

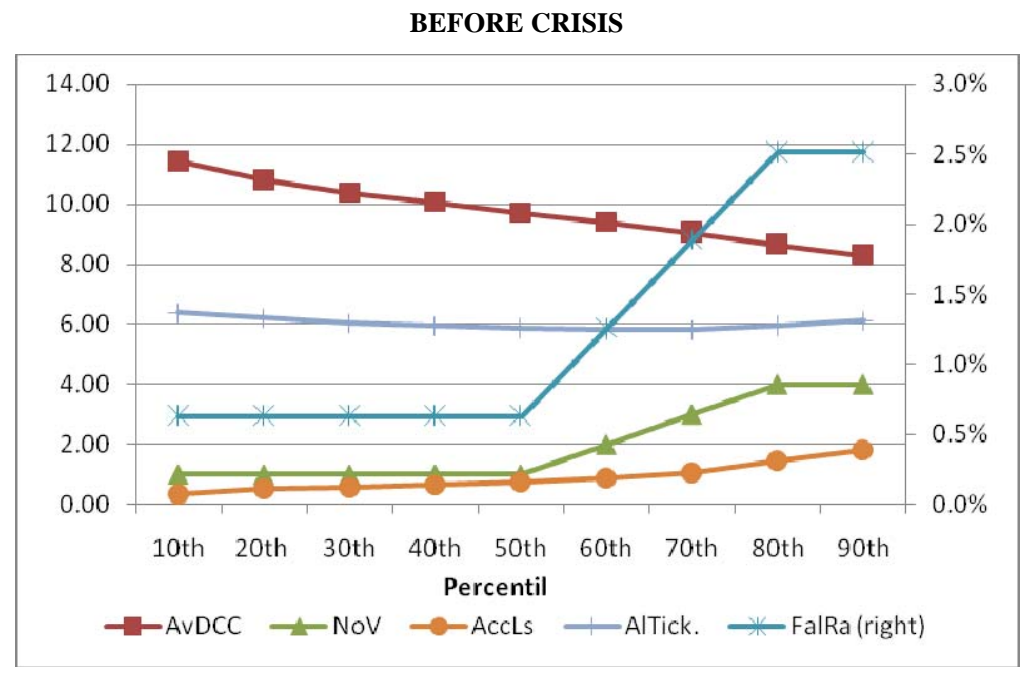

DURING CRISIS

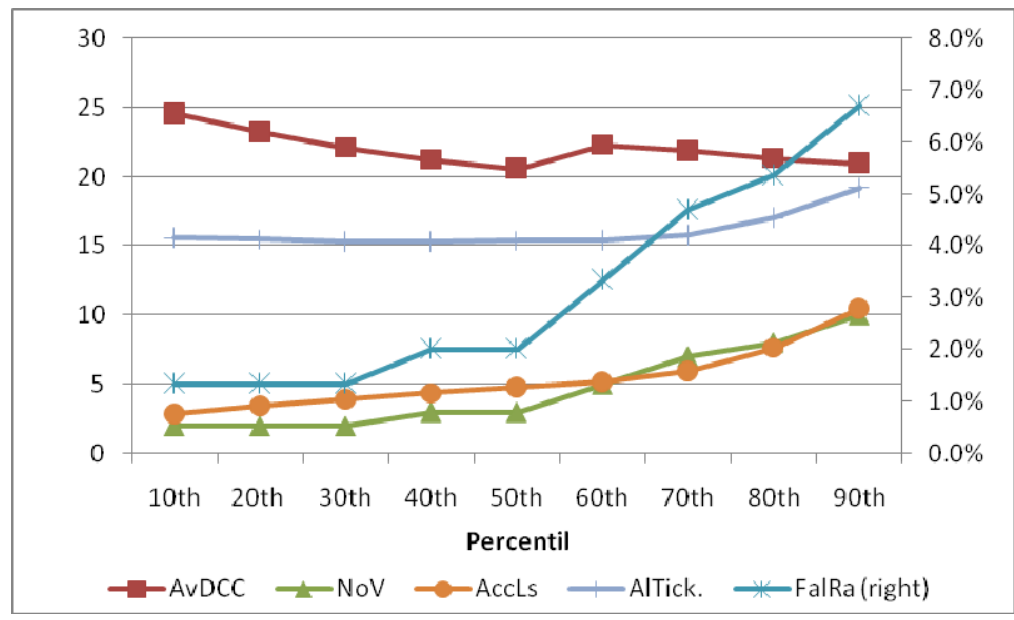

AFTER CRISIS

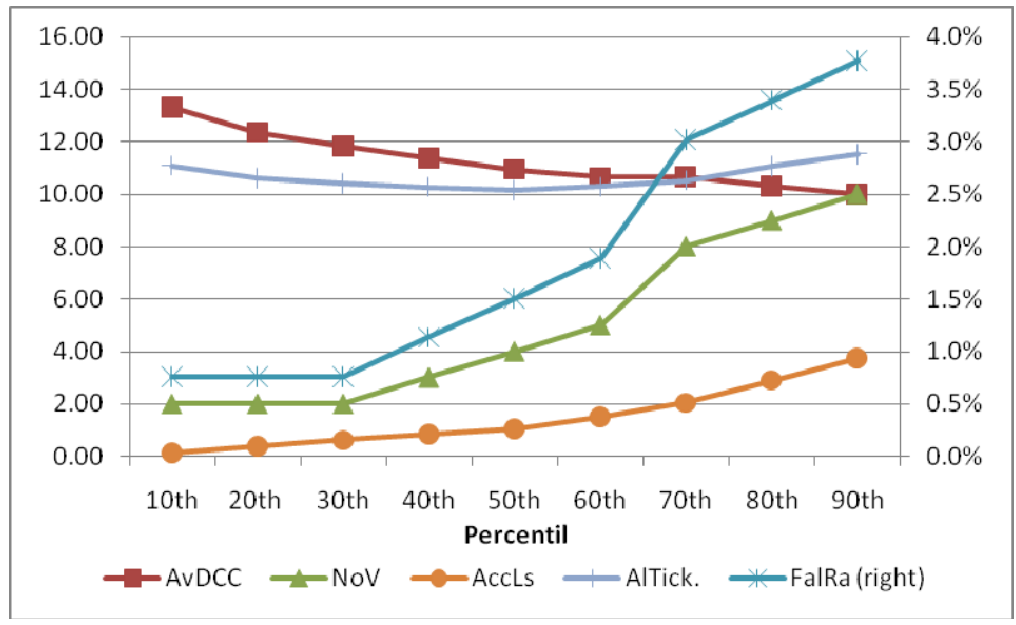


Figure 4. VaR for S\&P500 Returns

\section{January 2008 - 16 March 2010}

Peak_value Date Trough_value date

\begin{tabular}{lllll}
\hline S\&P Returns & 1305.323 & $11 / 8 / 2008$ & 676.5302 & $9 / 3 / 2009$
\end{tabular}

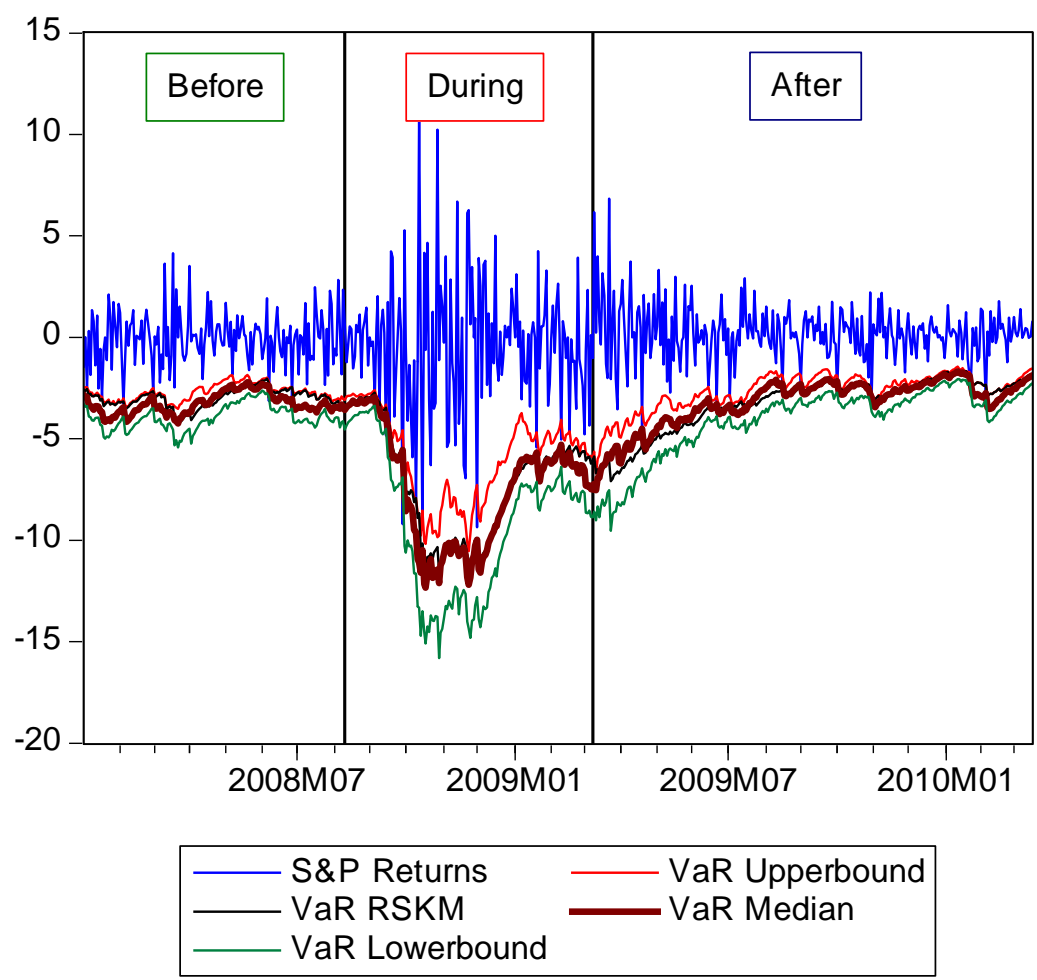

Note: The upper blue line represents daily returns for S\&P500 index. The upper red line represents the supremum, the lower green line the infinum, the bold brown line the median of the VaR forecasts of the alternative models (see Section 3), and the black line is the Riskmetrics VaR forecast. 
Figure 5. DCC and S\&P500 Returns

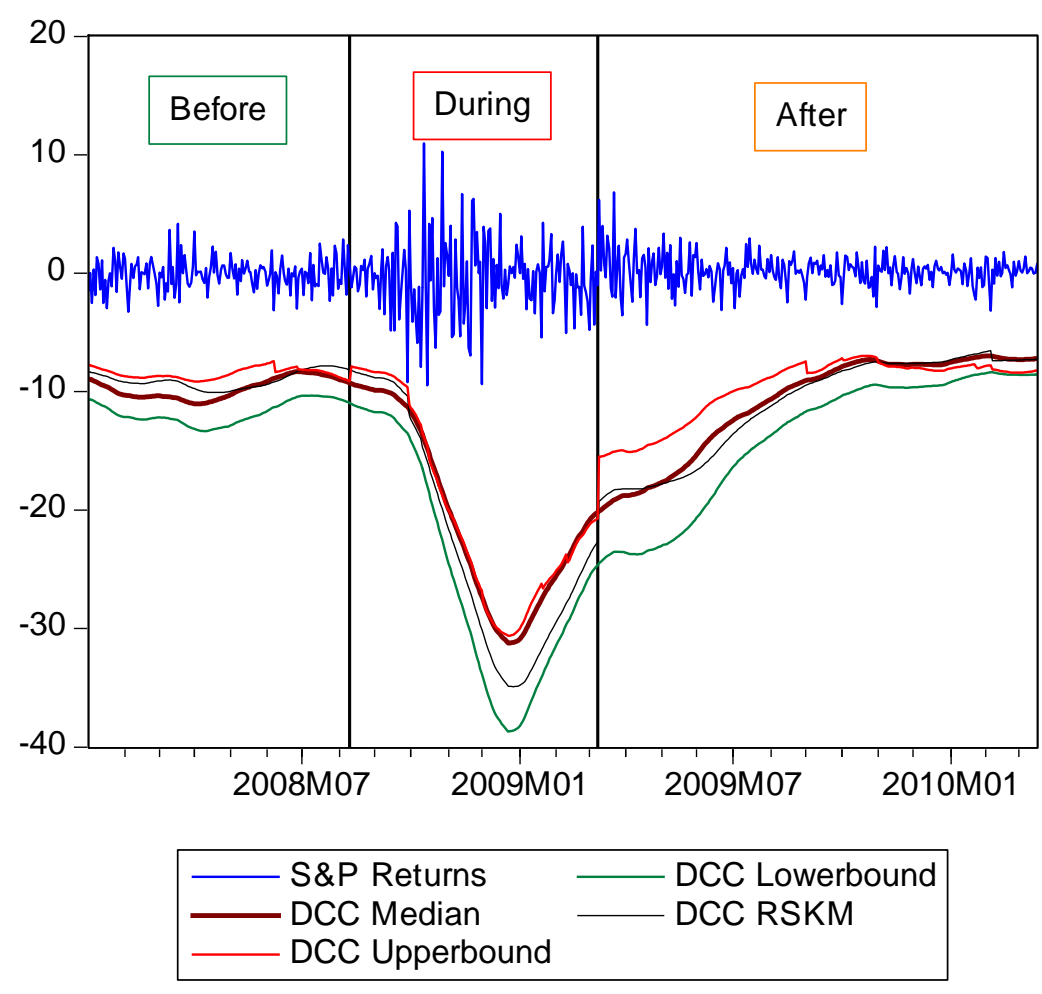


Table 1: Basel Accord Penalty Zones

\begin{tabular}{|l|c|c|}
\hline Zone & Number of Violations & $k$ \\
\hline Green & 0 to 4 & 0.00 \\
\hline Yellow & 5 & 0.40 \\
& 6 & 0.50 \\
& 8 & 0.65 \\
& 9 & 0.75 \\
& $10+$ & 0.85 \\
\hline Red & $\begin{array}{l}\text { Note: The number of violations is given for 250 business days. The } \\
\text { penalty structure under the Basel II Accord is specified for the } \\
\text { number of violations and not their magnitude, either individually or } \\
\text { cumulatively. }\end{array}$ \\
\hline
\end{tabular}


Table 2. Descriptive Statistics for S\&P500 Returns (\%)

3 January 2000 - 15 March 2010

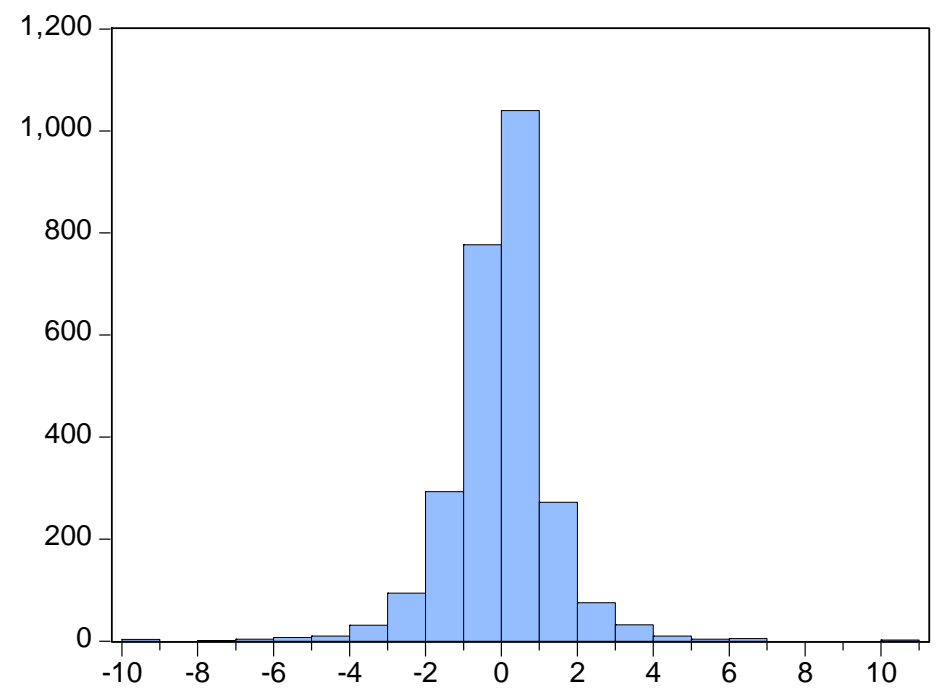

Observations 2660

Mean $\quad-0.009$

Median $\quad 0.008$

Maximum $\quad 10.96$

Minimum $\quad-9.470$

Std. Dev. $\quad 1.368$

Skewness $\quad-0.113$

Kurtosis $\quad 11.06$

Jarque-Bera 7209.7

Probability $\quad 0.0000$ 
Table 3. Comparing Alternative Models of Volatility

BEFORE CRISIS

DURING CRISIS

AFTER CRISIS

\begin{tabular}{|c|c|c|c|c|c|c|c|c|c|c|c|c|c|c|c|}
\hline \multirow[b]{2}{*}{ Model } & & & \\
\hline & AvDCC & NoV & FailRa & AcLoss & AlTick & AvDCC & NoV & FailRa & AcLoss & AlTick & AvDCC & NoV & FailRa & AcLoss & AlTick \\
\hline RSKM & 9.03 & 4 & $2.5 \%$ & 1.60 & 6.28 & 22.51 & 6 & $4.0 \%$ & 6.21 & 16.27 & 11.19 & 5 & $1.9 \%$ & 1.62 & 10.88 \\
\hline GARCH & 9.08 & 6 & $3.8 \%$ & 1.89 & 6.42 & 21.39 & 7 & $4.7 \%$ & 7.40 & 16.95 & 10.76 & 6 & $2.3 \%$ & 1.74 & 10.73 \\
\hline GJR & 9.00 & 3 & $1.9 \%$ & 1.03 & 5.75 & 20.11 & 4 & $2.7 \%$ & 5.16 & 15.53 & 10.71 & 8 & $3.0 \%$ & 2.75 & 11.10 \\
\hline EGARCH & 8.87 & 4 & $2.5 \%$ & 1.13 & 5.82 & 19.92 & 10 & $6.7 \%$ & 12.10 & 20.49 & 9.92 & 10 & $3.8 \%$ & 3.81 & 11.52 \\
\hline GARCH_t & 11.16 & 1 & $0.6 \%$ & 0.21 & 6.03 & 24.52 & 2 & $1.3 \%$ & 2.85 & 15.51 & 13.67 & 1 & $0.4 \%$ & 0.13 & 11.52 \\
\hline GJR_t & 10.80 & 1 & $0.6 \%$ & 0.57 & 6.26 & 24.27 & 2 & $1.3 \%$ & 2.88 & 15.57 & 12.21 & 3 & $1.1 \%$ & 0.56 & 10.47 \\
\hline EGARCH_t & 10.75 & 1 & $0.6 \%$ & 0.48 & 6.19 & 20.95 & 2 & $1.3 \%$ & 4.51 & 15.23 & 11.14 & 4 & $1.5 \%$ & 0.80 & 9.99 \\
\hline GARCH_g & 9.81 & 2 & $1.3 \%$ & 0.79 & 5.90 & 22.11 & 5 & $3.4 \%$ & 4.41 & 15.35 & 11.94 & 2 & $0.8 \%$ & 0.73 & 10.75 \\
\hline GJR_g & 9.82 & 1 & $0.6 \%$ & 0.80 & 5.96 & 21.97 & 3 & $2.0 \%$ & 3.96 & 15.37 & 11.08 & 4 & $1.5 \%$ & 1.34 & 10.39 \\
\hline EGARCH_g & 9.75 & 1 & $0.6 \%$ & 0.72 & 5.89 & 19.58 & 6 & $4.0 \%$ & 7.10 & 16.68 & 10.20 & 6 & $2.3 \%$ & 2.22 & 10.56 \\
\hline Inf & 11.78 & 1 & $0.6 \%$ & 0.21 & 6.42 & 25.26 & 2 & $1.3 \%$ & 2.64 & 15.78 & 13.99 & 1 & $0.4 \%$ & 0.07 & 11.56 \\
\hline Sup & 8.45 & 6 & $3.8 \%$ & 2.06 & 6.28 & 20.01 & 11 & $7.4 \%$ & 12.28 & 20.52 & 9.71 & 10 & $3.8 \%$ & 4.31 & 11.89 \\
\hline Mean & 9.77 & 1 & $0.6 \%$ & 0.69 & 5.82 & 20.73 & 3 & $2.0 \%$ & 4.57 & 15.21 & 11.11 & 3 & $1.1 \%$ & 0.99 & 10.21 \\
\hline $10^{\text {th }}$ Per. & 11.43 & 1 & $0.6 \%$ & 0.34 & 6.38 & 24.56 & 2 & $1.3 \%$ & 2.87 & 15.62 & 13.34 & 2 & $0.8 \%$ & 0.13 & 11.10 \\
\hline 20th Per. & 10.81 & 1 & $0.6 \%$ & 0.51 & 6.21 & 23.21 & 2 & $1.3 \%$ & 3.49 & 15.49 & 12.39 & 2 & $0.8 \%$ & 0.40 & 10.60 \\
\hline 30th Per. & 10.37 & 1 & $0.6 \%$ & 0.56 & 6.03 & 22.07 & 2 & $1.3 \%$ & 3.96 & 15.34 & 11.85 & 2 & $0.8 \%$ & 0.62 & 10.40 \\
\hline 40th Per. & 10.06 & 1 & $0.6 \%$ & 0.65 & 5.94 & 21.23 & 3 & $2.0 \%$ & 4.40 & 15.32 & 11.38 & 3 & $1.1 \%$ & 0.85 & 10.27 \\
\hline $\begin{array}{l}50^{\text {th }} \text { Per. } \\
\text { (Median) }\end{array}$ & 9.71 & 1 & $0.6 \%$ & 0.76 & 5.86 & 20.57 & 3 & $2.0 \%$ & 4.81 & 15.37 & 10.95 & 4 & $1.5 \%$ & 1.07 & 10.14 \\
\hline 60th Per. & 9.39 & 2 & $1.3 \%$ & 0.87 & 5.80 & 22.29 & 5 & $3.4 \%$ & 5.21 & 15.41 & 10.66 & 5 & $1.9 \%$ & 1.51 & 10.29 \\
\hline 70th Per. & 9.06 & 3 & $1.9 \%$ & 1.05 & 5.80 & 21.86 & 7 & $4.7 \%$ & 6.00 & 15.84 & 10.68 & 8 & $3.0 \%$ & 2.05 & 10.53 \\
\hline 80th Per. & 8.65 & 4 & $2.5 \%$ & 1.45 & 5.95 & 21.32 & 8 & $5.4 \%$ & 7.66 & 17.04 & 10.32 & 9 & $3.4 \%$ & 2.90 & 11.06 \\
\hline $90^{\text {th }}$ Per. & 8.28 & 4 & $2.5 \%$ & 1.81 & 6.11 & 20.97 & 10 & $6.7 \%$ & 10.53 & 19.19 & 10.01 & 10 & $3.8 \%$ & 3.75 & 11.54 \\
\hline
\end{tabular}

Note: Percentage of Days Minimizing Daily Capital Charges (\%DmDCC), Average of Daily Capital Charges (AvDCC), Number of Violations (NoV), Failure Rate (FailRa), Accumulated Loses (AcLoss), and Asymmetric Linear Tick loss function value (AlTick) for alternative models of volatility. 
Table 4. Quartiles for Evaluation Statistics

\begin{tabular}{|c|c|c|c|c|c|c|c|c|c|c|c|c|c|c|c|}
\hline & & Before & & & & & & During & & & & & After & & \\
\hline Quartiles & AvDCC & NoV & FailRa & AcLoss & Altick & DCC & NoV & FailRa & AcLoss & Altick. & DCC & NoV & FailRa & AcLoss & Altick. \\
\hline 1 & 9.06 & 1.0 & $0.6 \%$ & 0.65 & 5.88 & 20.34 & 2.5 & $1.7 \%$ & 4.19 & 15.37 & 10.74 & 3.5 & $1.3 \%$ & 0.77 & 10.43 \\
\hline 2 & 9.75 & 1.0 & $0.6 \%$ & 0.79 & 5.96 & 21.39 & 4.0 & $2.7 \%$ & 4.81 & 15.53 & 11.08 & 4.0 & $1.5 \%$ & 1.34 & 10.73 \\
\hline 3 & 10.28 & 3.5 & $2.2 \%$ & 1.08 & 6.23 & 22.31 & 6.0 & $4.0 \%$ & 6.65 & 16.48 & 11.56 & 6.0 & $2.3 \%$ & 1.98 & 10.99 \\
\hline \multicolumn{16}{|l|}{ Models } \\
\hline RSKM & 1 & 4 & 4 & 4 & 4 & 4 & 4 & 4 & 3 & 3 & 3 & 3 & 3 & 3 & 3 \\
\hline EGARCH_t & 4 & 1 & 1 & 1 & 3 & 2 & 1 & 1 & 2 & 1 & 3 & 2 & 2 & 2 & 1 \\
\hline Median & 2 & 1 & 1 & 2 & 1 & 2 & 2 & 2 & 2 & 1 & 2 & 2 & 2 & 2 & 1 \\
\hline
\end{tabular}

Note: The top part of the table gives the value of the $r$ th quartile for the evaluation statistics in Table 3 when considering all the GARCH-family models and

Riskmetrics. The bottom part of the table is the quartile where the evaluation statistics fell for the RSKM, EGARCH_t and Median models. For example, for the RSKM model, an entry of 'one' in the first column means that the corresponding AvDCC fell in the first quartile. 